\title{
Iris Recognition based on Block Theory and Self-adaptive Feature Selection
}

\author{
Jia Zhen Liang \\ Software Technology Vocational College, North China University of Water \\ Resources and Electric Power, Zhengzhou 450045, China \\ 1989643460@qq.com
}

\begin{abstract}
In order to improve the performance of iris recognition, a novel method for iris recognition based on block theory and self-adaptive feature selection is proposed in this paper. Firstly, the normalized iris image is decomposed by convolving with multi-scale and multi-orientation Gabor filters, and then separated into several blocks, the block feature vector which includes mean and variance of Gabor coefficients inside each block can be obtained through statistical techniques, the iris feature vector of the whole iris image is then constructed by conjugating the block feature vector in row column order, finally the two-classifier of iris image are established based on the most distinguishable features, and the multi-classifiers of iris image are established by voting mechanism, and the performance is test by CASIA iris database. The results show that, compared with the traditional iris recognition methods, the proposed method has improved the iris recognition rate.
\end{abstract}

Keywords: iris recognition, Gabor filter, block statistical, feature extraction, vote

\section{Introduction}

With the rapid development of the Internet and the frequent information exchange, personal identification and information protection have become increasingly important. Iris has advantages of non-contact, uniqueness, high reliability, stability and difficulty to counterfeit, and iris recognition has a huge potential market in the military, public security, civil and other fields. Therefore, iris recognition becomes a heatedly discussed topic in the current biological recognition [1].

In the process of iris recognition, it is crucial to identify the important feature of iris categories from iris images. Wildes, et al., put forward the iris feature extraction algorithm of Laplacian pyramid decomposition, and recognized the iris through Fisher linear transformation; Boles et al adopted three sample wavelets to extract iris recognition features [2, 3]. Gabor filter is a powerful tool for texture analysis. Since 1993, Daugman proposed to extract iris features by using two-dimensional Gabor and adopt Hamming distance iris recognition algorithm. It has high recognition performance, which is the core algorithm of current domestic and overseas commercial iris recognition system, and has become the mainstream technology in present iris recognition [4]. The algorithm filters the iris images by using two-dimensional Gabor filter under multiple frequencies, so as to extract the iris texture features at different frequencies. On this basis, many scholars have made in-depth researches, Yuan Weiqi, et al., proposed an iris recognition algorithm based on single-frequency two-dimensional Gabor filter, found the optimal parameters from lots of frequency parameters, improved the feature extraction rate, and reduced the storage space [5]; Yin Yong et al., put forward the iris recognition algorithm based on cross power spectrum, that is, found out the filter code corresponding to the maximum feature amplitude value for code identification [6]; Karen, et al., proposed a simple iris recognition algorithm to identify the stability of Gabor phase features, they considered 
that if the real or imaginary Gabor features got close to the real or imaginary axis (small value), Gabor phase features at the position would be unstable and treated as noise points, which would not participate in the feature matching. As a result, the inter-class Hamming distance was increased and the recognition rate was improved [7]. Currently, Gabor wavelet analysis is often directly made towards the whole iris image. However, owing to multi-scale multi-directional magnifying glass effect of the two-dimensional Gabor transform, the iris image feature dimension increases sharply, inevitably resulting in the high dimensionality of iris features vector as well as failure of direct classification and identification [8]. For this reason, some scholars have proposed iris recognition algorithm with integration of Gabor and Fisher discriminant. The algorithm better compresses the iris feature dimension, but misses a lot of effective features, resulting in decreased subsequent iris recognition rate [9]. How to effectively reduce the dimension of Gabor features has become the key to solving the problem of iris recognition [10].

Considering high dimensionality of directly extracting iris feature through Gabor wavelet, this paper proposes an iris recognition algorithm based on block idea and self-adaptive feature selection (Gabor-FS). First of all, the study divides the iris image into blocks, and respectively extracts the statistics of Gabor features; then according to the feature resolution size, this paper selects the optimal iris feature subset to build two classifiers, and finally builds iris multiple classification classifiers via the voting mechanism, so as to use CASIA iris image database for performance test and verify the effectiveness of the algorithm.

\section{Iris Preprocessing}

In addition to the iris information, the human eye images captured by the image capture devices also include eyelashes, eyelids, light spots and other noises [12]. In order to accurately extract the iris information and eliminate the adverse effects of noises, this paper conducts preprocessing of inner and outer edge localization, eyelid localization, normalization, noise shield template generation, and contrast enhancement towards iris images.

\subsection{Inner and Outer Edge Localization of Iris}

Inner and outer edge of the iris can be contemplated with a round. The localization of inner and outer edge aims at determining the centers and radium of two circles. First of all, rough localization shall be conducted towards the center and radius of the edge and inner and outer edge of the iris shall be accurately localized. Rough localization of inner edges can restrict the searching scope of inner and outer edge to a relatively small area, greatly saving the overall localization time. After determining the parameters of inner edge rough localization, this paper conducts accurate localization for inner and outer edge by using the circle detection operator Equation (1). Figure 1 (a) shows the iris image after inner and outer edge localization.

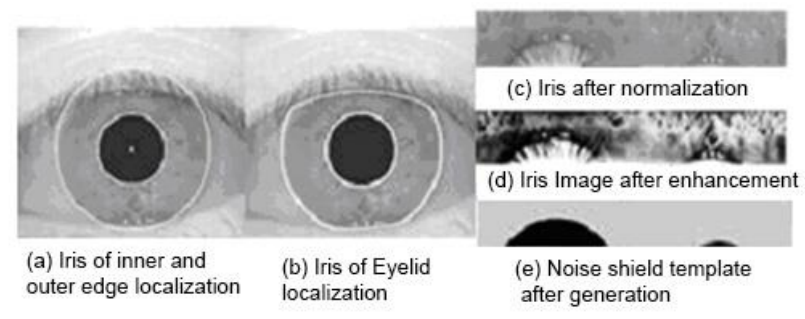

Figure 1. CASIA Database Iris Image Preprocessing 


\subsection{Eyelid localization}

The collected iris is often blocked by eyelid to a certain degree. By localizing the eyelid edge, it is possible to accurately extract iris information, eliminate the interference eyelid occlusion, and generate the iris noise shield template by using the eyelid localization results. The eyelid can be approximately described by a quadratic curve. The image after eyelid localization is shown in Figure 1 (b).

\subsection{Iris Normalization and Noise Shield Template Generation}

Iris size varies with people. Under different collection conditions, the same irises are not the same. To eliminate the inconvenience brought by such differences to recognition, it is necessary to be normalized and expand the iris region to uniform size. Figure 1 (c) is the normalized iris image. According to Figure 1 (c), the normalized iris image contains both iris information and noise information. To avoid the interference caused by noise in the recognition process, the proposed solution algorithm is to generate noise shield template. The template is the same and corresponds with the size of normalized iris image. The template marks the iris information as 1 and marks the noise as 0 . Noises incurred by eyelid occlusion are marked according to the results of the above eyelid localization. For example, Figure 1 (e) shows the generated noise shield template, and the darker part corresponds to the noise caused by eyelid occlusion. When matching the iris feature code, the noise interference can be effectively removed by introducing noise shield template.

\subsection{Contrast Enhancement}

Due to the impacts of collection devices and environmental factors, the collected iris images tend to have lower contrast. Histogram equalization algorithm is used to enhance the normalized iris image, and the enhancement effects are shown in Figure 1 (d).

\section{Iris Feature Extraction}

\subsection{Gabor transform}

Gabor can better simulate the contour of single cell receptive field in the brain and capture the prominent visual attributes. In particular, Gabor wavelet extracts the multi-scale multi-directional spatial frequency features within a specific area of images and zooms the gray scale changes like microscope. Since Gabor features are locally extracted from the image, so they have good robustness towards the light changes, iris zoom and reflection of the eye surface ${ }^{[13]}$. Two-dimensional Gabor kernel function is defined as:

$$
\psi_{\mu, v}(z)=\frac{\left\|k_{u, v}\right\| 2}{\sigma^{2}} e^{-\frac{\left\|k_{u, v}\right\| 2\|z\| 2}{2 \sigma^{2}}} \cdot\left(e^{i k_{\mu, v} \cdot z}-\mathrm{e}^{-\frac{\sigma^{2}}{2}}\right)
$$

Where, $\sigma$ defines the wavelet filter bandwidth; $i$ is the complex operator; $z=(x, y)$ represents the pixel coordinates; $\mu, v$ respectively stand for the wavelet direction and scale. In $k_{v}=k_{\max } / f^{v}, k_{\max }$ represents the maximum frequency, and $f$ stands for the interval factors. $k_{\mu, v}=k_{v}^{i \phi_{\mu}}$ stands for the wavelet vector.

Gabor feature extraction is completed by Gabor filter with v scales, $\mu$ directions through convolution operations. Given $I(Z)$ is the pixel value at the iris image point $z$, the convolution with the Gabor filter is defined as:

$$
G_{\mu, v}(z)=\psi_{\mu, v}(z) * I(z)
$$


Where, $\mathrm{G}_{\mu, v}(z)$ is the filter result of scale $\mu$ direction wavelet kernel function at the point $\mathrm{z}$.

After Gabor kernel function convolution towards each point $P(m, n)$ on the image, Gabor feature dimension is quite high. For example, roughly make statistics for a $50 \times 50$ picture according to the pixel gray scale, there are 2500 dimensions of features, which in itself belong to high dimension. If Gabor filters with five scales and 8 directions, the original feature number increases to $5 \times 8 \times 50 \times 50=100000$ dimensions after Gabor transform. Obviously, such a high-dimensional features vector is not suitable for direct iris recognition. Therefore, appropriate steps must be taken for feature dimension reduction.

\subsection{Gabor Coefficient Block Ideas}

Since the original Gabor features have high dimensionality, redundancy and other issues, this paper first divides the iris area into blocks, makes statistics for each sub-block's Gabor coefficient means and variances at specific scales and directions, combines these means and variances to form the block features vector (BFV) in the manner of scale before direction, and finally splices these block features vector BFV to form the iris features vector (IFV) in the manner of line before column, so as to effectively reduce the feature dimension and make full use of the statistical information of all Gabor coefficients in the block. Its construction process is shown in Figure 2.

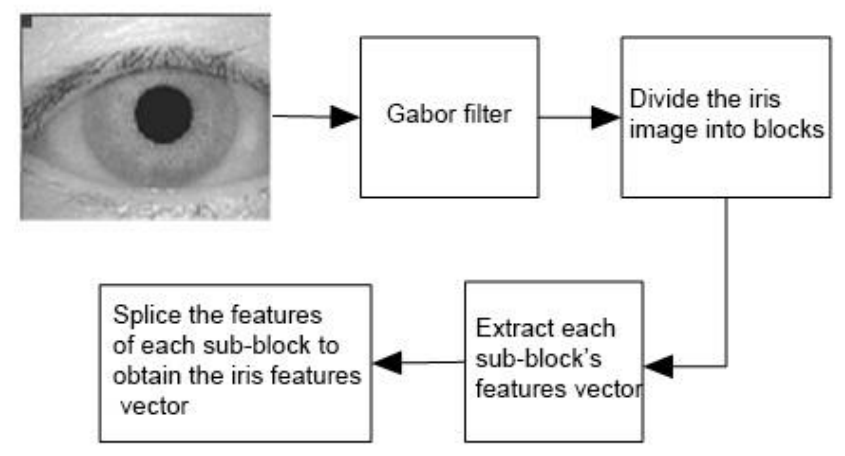

\section{Figure 2. Schematic Diagram of Gabor Coefficient Features Block Extraction}

\subsection{Extracting Features of Each Block}

Divide the iris region into blocks according to specific length and width, and get the Block to be processed. The number of all points in the block is $N$, so each block's feature extraction is finished by following steps:

(1) Statistics on the filtering results' mean of all points at $c$ scale and $f$ direction in the Block:

$$
\bar{G}_{c, f}=\frac{1}{n} \sum_{t \in \text { Block }} G_{c, f}(t)
$$

(2) Statistics on the filtering results' standard deviation of all points at $c$ scale and $f$ direction in the Block

$$
D_{c, f}=\sqrt{\frac{\sum_{t \in \text { Block }}\left(G_{c, f}(t)-\bar{G}_{c, f}\right)^{T}\left(G_{c, f}(t)-\bar{G}_{c, f}\right)}{N-1}}
$$

(3) Block's features vector BFV can be expressed by vector $V_{\text {Block }}$ :

\subsection{Spliced Formation of Iris Image Features Vector}

Assumed the iris image is divided into $P \times Q$ blocks, the block features vector at the $p$-th line and $q$-th column is expressed as $V_{p, q}, p=1,2, \ldots, P, q=1,2, \ldots, \mathrm{Q}$, then the iris image 
features vector IFV can be expressed by the vector $V_{\text {Iris: }}$ :

$$
\left.V_{\text {Block }}=\left\|\bar{G}_{0,0}\right\|,\left\|\bar{G}_{0,1}\right\|, \cdots,\left\|\bar{G}_{4,7}\right\|, D_{0,0}, D_{0,1}, \cdots, D_{4,7}\right)
$$

When using Gabor filter with 5 scales and 8 directions, $V_{\text {Iris }}$ is a $P \times Q \times 80$-dimensional features vector.

\section{Classifier Design}

It is used to describe the Gabor feature's filtering results of iris image from the filters at multiple frequencies and multiple directions in different positions. However, the researchers have inadequate priori knowledge in determining which features are more beneficial for the specific task of iris recognition. This paper adopts the voting mechanism to combine multiple binary classifiers into a multi-classifier. Meanwhile, when building the binary classifiers, the paper introduces the self-adaptive feature selection mechanism and selects the subset $B F V$ from iris features vector with the most powerful resolution ability. Iris classifier is built as shown in Figure 3.

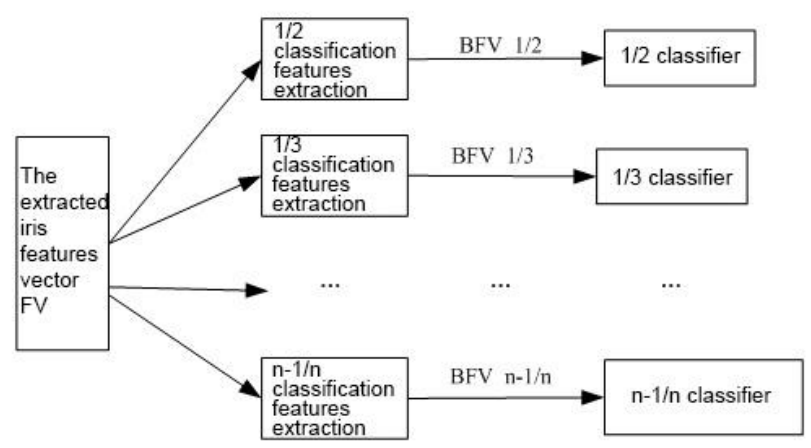

Figure 3. Schematic Diagram of Iris Classifier

\section{a. Construction of Binary Classifier}

Take A/B binary classifier as an example, two types of training samples were respectively of $n_{\mathrm{A}}$ and $n_{\mathrm{B}}$, the training set were marked as: $C_{A}=\left\{s_{A, 1}, S_{A, 2}, \cdots, S_{A, n_{A}}\right\}$, $C_{B}=\left\{S_{B, 1}, S_{B, 2}, \cdots, S_{B, n_{B}}\right\}$, where, the training sample is $s_{i, j}$ with the initial dimension of $h$, namely $S_{i, j}=\left\{s_{i, j, 1}, s_{i, j, 2}, \cdots, s_{i, j, 1 h}\right\}^{T}$.

Introduce the resolution ability $R_{\mathrm{A}, \mathrm{B}, \mathrm{K}}(1 \leq k \leq h)$ indicator to express feature's discrimination over $\mathrm{A} / \mathrm{B}$ binary classifier at the $k$-th dimension, and $R_{A, B, K}$ can be calculated by Formula (7):

$$
R_{A, B, k}=\frac{\left(\mu_{A, k}-\mu_{B, k}\right)^{2}}{\sigma_{A, k}^{2}+\sigma_{B, k}^{2}}
$$

In the formula, $\mu_{i, k}$ and $\sigma_{i, k}^{2}$ respectively represent the means and variances of all training samples of $i$ class features at the k-th dimension, which is expressed in below Formula (8): 


$$
\left\{\begin{array}{l}
\mu_{i, k}=\frac{1}{n_{i}} \sum_{j=1}^{n_{i}} S_{i, j, k} \\
\sigma_{i, k}^{2}=\frac{1}{n_{i}-1} \sum_{j=1}^{n_{i}}\left(S_{i, j, k}-\mu_{i, k}\right)^{2}
\end{array}\right.
$$

Based on $\mathrm{A} / \mathrm{B}$ binary training samples, the resolution of $\mathrm{A} / \mathrm{B}$ binary classifier in iris features vector $I F V$ can be calculated by formula (7). Take $m$ dimension with the largest resolution to compose the optimal features subset $B F V$.

\section{b. Multiple Classifiers Construction by Using the Voting Mechanism}

Assumed there are $\omega$ people in the database, the number of classifiers to be built is $C_{\omega}^{2}=\omega(\omega-1) / 2$. For the iris features vector $u$ to be judged, it shall be determined by all classifiers and determine the attribution of $u$ by using the voting mechanism. For example, if $\mathrm{A} / \mathrm{B}$ classifier determines that $u$ belongs to Class $\mathrm{A}$, Class $\mathrm{A}$ gets one vote and Class $\mathrm{B}$ gets no vote. When all $C_{\omega}^{2}$ classifiers finish the determination, the class with the most votes is the class that $u$ belongs to, which is formally expressed as follows:

$$
u \in i, \text { if } \lambda(i, u)=\max _{j=1,2, \cdots, \omega} \lambda(i, u)
$$

Where:

$$
\begin{array}{r}
\lambda(i, u)=\sum_{\eta=1}^{\omega(\omega-1) / 2} \chi(\eta, j, u) \\
\chi(\eta, j, u)=\left\{\begin{array}{l}
1, \text { if } \eta(u)=j \\
0, \text { otherwise }
\end{array}(11)\right.
\end{array}
$$

In the formula, $\eta(u)$ represents the classification results of classifier $\eta$ over $u$; $\lambda(i, u)$ represents the votes obtained from the $i$-th class in $u$ classification process.

\section{Simulation Test}

\section{a. Data Sources}

The paper takes iris images from the CASIA 1.0 and CASIA 2.0 iris database as the test object, which is an iris image sample database freely provided by key national laboratory's iris recognition research group of Institute of Automation, Chinese Academy of Sciences. It is the largest shared iris database in the world, specially used for iris recognition. Part of the iris image is shown in Figure 4 [14]. Gabor filter parameters are set to be: 5 scales $(v \in\{0,1,2,3,4\}), 8$ directions $(\mu \in\{0,1, \ldots, 7\}), \sigma=2 \pi, k \max =\pi / 2$, $f=\sqrt{2}$. In order to make the results of Gabor-FS algorithm's iris recognition result comparable, this paper selects the Gabor filters (Gabor) without division into blocks, principal component analysis (PCA), PCA + locality preserving projection algorithm (PCA+LPP), two-dimensional principal component analysis (2DPCA) and two-dimensional locality preserving projection algorithm (2DLPP) for feature extraction and selection of comparative experiments. To facilitate processing, the iris image is adjusted to $50 \times 50$ pixels. The test experiment is conducted under the CPU of Intel core2 3.0Ghz, the 2G RAM hardware environment and the Matlab 2012 software environment. 

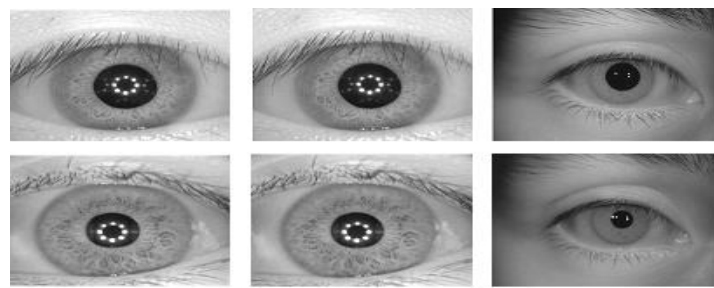

Figure 4. Part of CASIA's Iris Image

\section{b. CASIA 1.0 Database}

\section{i. Comparison of Iris Recognition Rate}

In CASIA 1.0 iris database, the image size is $280 \times 320$ pixels with iris images for 108 eyes, 7 images for each eye and a total of 756 images. It uses contacted device to shoot, which is less affected by the light and eyelash blocking. Randomly select 5 images as the training set for each eye, the rest images are used as the test set, and the recognition rates for each feature extraction algorithms are shown in Table 1. According to Table 1, in the same experimental environment, Gabor-FS iris feature extraction can reflect the iris image class information more accurately and comprehensively, and help improve the iris recognition rate. The average recognition rate is up to $97.55 \%$, much higher than $87.14 \%$ of PCA algorithm, 91.38\% of PCA+LPP algorithm, 91.50\% of 2DPCA algorithm, 92.17\% of 2DLPP algorithm, indicating that the presented iris feature extraction idea is correct. Compared with the traditional Gabor algorithm, Gabor-FS selection features dimension is $1 / 12$ of the traditional Gabor algorithm, the final iris recognition features keep about 800 dimensions, which reduce two magnitude orders compared with the original 10,000 dimensions of iris features. As a result, the time-consuming complexity of iris recognition has been greatly reduced, Gabor-FS average iris recognition rate reaches about 13 times of the traditional Gabor algorithm, which improves the iris recognition speed and efficiency, fully meets the real-time recognition of iris images, and significantly improves the iris recognition rate, so the proposed iris recognition algorithm is practicable and superior.

Table 1. Comparison of CASIA 1.0 Different Feature Extraction Algorithms' Recognition Rates

\begin{tabular}{cccc}
\hline $\begin{array}{c}\text { Feature } \\
\text { Extraction } \\
\text { Algorithm }\end{array}$ & $\begin{array}{c}\text { Optimal } \\
\text { Feature } \\
\text { Dimensi } \\
\text { on }\end{array}$ & $\begin{array}{c}\text { Maximum } \\
\text { Iris } \\
\text { Recognition } \\
\text { Rate }(\%)\end{array}$ & $\begin{array}{c}\text { Average } \\
\text { Recognition } \\
\text { Time of Each } \\
\text { Test Sample (ms) }\end{array}$ \\
\hline PCA & 60 & 87.14 & 20.8 \\
PCA+LPP & 56 & 91.38 & 28.5 \\
2DPCA & 125 & 91.50 & 27.2 \\
2DLPP & 125 & 92.17 & 23.3 \\
Gabor & 10000 & 95.13 & 400.1 \\
Gabor-FS & 800 & 97.55 & 30.2 \\
\hline
\end{tabular}

\section{ii. Effects of the sub-block size on Recognition Rate}

Iris images are divided into sub-blocks with different sizes, and the dimensions of iris features vector $I F V$ are not the same, as shown in Table 2. When the block differs in sizes, the iris recognition rate curve is shown in Figure 5. 
Table 2. Block Number and Dimension Size for Different Block Sizes

\begin{tabular}{|c|c|c|}
\hline $\begin{array}{l}\text { Block } \\
\text { Size }\end{array}$ & $\begin{array}{c}\text { Block } \\
\text { Number }\end{array}$ & FFV's Dimension \\
\hline $2 \times 2$ & $25 \times 25=625$ & $25 \times 25 \times 80=50000$ \\
\hline $5 \times 5$ & $10 \times 10=100$ & $10 \times 10 \times 80=8000$ \\
\hline $10 \times 10$ & $5 \times 5=25$ & $5 \times 5 \times 80=2000$ \\
\hline $25 \times 25$ & $2 \times 2=4$ & $2 \times 2 \times 80=320$ \\
\hline
\end{tabular}

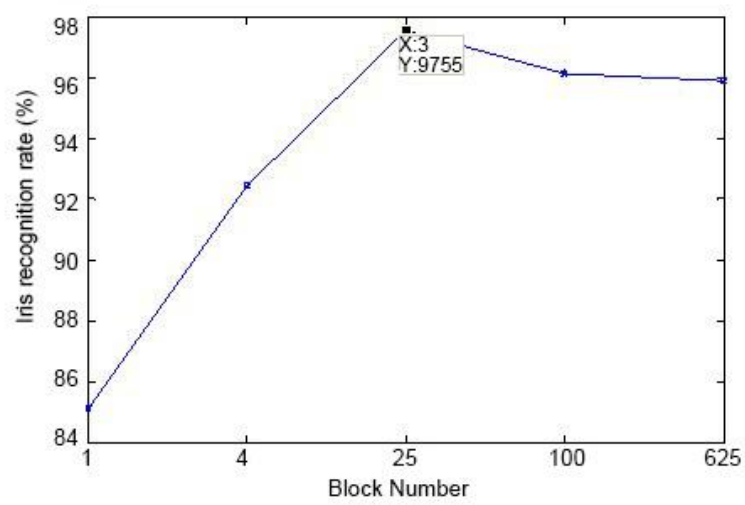

Figure 5. Changing Curve between Block Numbers and Iris Recognition Rate

According to Figure 5, as the number of blocks increases, the iris recognition rate gradually increases, which is mainly because that the block size can be deemed as the processing particle size of iris image's original Gabor coefficients. The larger the block is, the coarser the processing particle size is. The statistical information will lose a lot of details in the block, affecting the recognition accuracy and unable to effectively characterize the iris. When reaching $10 \times 10$, the iris recognition rate can reach a maximum value. At this time, the iris local feature information can be fully highlighted. If the block number continues to increase, it has small impacts on the recognition rate, and redundant phenomenon occurs between features, which will extend the recognition time. When fully exploiting the iris image details, it effectively processes the original Gabor coefficients, reduces the dimensions of iris image features vector $I F V$. In this paper, the optimal block size is $10 \times 10$ pixels and the block number is $10 \times 10=100$.

\section{iii. Feature Dimension Effects on Iris Recognition Performance}

When the optimal block size is $10 \times 10$ pixels, the iris image is divided into $10 \times 10=100$ pieces. Through block statistics, the dimension of iris features vector is $21 \times 10 \times 80=8000$ dimensions. The relationship between the feature dimensional size and iris recognition rate is shown in Figure 3.



Figure 6. Changing Curve between Feature Dimension and Recognition Rate 
According to Figure 6, when the feature dimension is small, iris recognition will be relatively low, which is because that lesser vectors carry insufficient amount of information and cannot accurately describe the iris category information. As the feature dimension increases, the iris recognition rate will slowly increase. When the feature dimension reaches 800 , the iris recognition rate will reach the maximum $(97.55 \%)$ and the recognition rate will be basically stable, indicating that the iris feature with the most expressive ability has been extracted at the time. If the feature dimension continues to increase, redundancy occurs between features, unnecessary interference is introduced, which will not only increases the computation amount, but also results in over-fitting data and decreased iris recognition rate, so the optimal feature dimension is selected to be 800 . Note: Figure 6 shows that the iris recognition rate with multiple features vectors (such as $1000,4400,7000)$ is above $95 \%$, exceeding the recognition rate of comparison algorithm. It means Gabor-FS iris recognition algorithm has better flexibility and robustness.

\section{c. CASIA 2.0 database}

CASIA 2.0 iris image database is a type of 8-bit grayscale $480 \times 640$ iris image, collects iris images for 60 eyes, 20 images for each eye and a total of 1200 images. It uses non-contacted device to shoot, which is greatly affected by the uneven illumination and eyelash occlusion. Randomly select 5 images for each eye, 300 images are used as independent test samples, and the rest are used as training samples. The average iris recognition rates of all algorithms are shown in Table 3. According Table 3, the iris recognition rate of Gabor-FS algorithm is higher than the comparison algorithm, and the comparative results prove that Gabor-FS has better solved the problems in the current iris recognition process.

\section{Table 3. Comparison of CASIA 2.0 Different Features Extraction Algorithms'} Recognition Rate

\begin{tabular}{cccc}
\hline $\begin{array}{c}\text { Feature } \\
\text { Extraction } \\
\text { Algorithm }\end{array}$ & $\begin{array}{c}\text { Optimal } \\
\text { Feature } \\
\text { Dimension }\end{array}$ & $\begin{array}{c}\text { Maximum } \\
\text { Iris } \\
\text { Recognition } \\
\text { Rate }(\%)\end{array}$ & $\begin{array}{c}\text { Average } \\
\text { Recognition Time } \\
\text { of Each Test } \\
\text { Sample }(\mathrm{ms})\end{array}$ \\
\hline PCA & 58 & 82.33 & 14.0 \\
PCA+LPP & 55 & 87.40 & 16.3 \\
2DPCA & 120 & 88.77 & 21.9 \\
2DLPP & 120 & 89.05 & 20.5 \\
Gabor & 10000 & 93.05 & 415.7 \\
Gabor-FS & 545 & 95.23 & 26.1 \\
\hline
\end{tabular}

\section{d. Performance Comparison with Existing Iris Recognition Algorithms}

To make the results of Gabor-FS algorithm be more convincing, this paper adopts the classical iris recognition algorithms Wilds, Boles, Daugman and literature [15] to conduct the comparative experiments towards CASIA 1.0, 2.0 iris dataset under the same conditions. All algorithms are implemented through Matlab 2012 self-programming. The average iris recognition rate of the algorithm is shown in Figure 7. According to Figure 7, the average iris recognition rate of Gabor-FS algorithm is higher than the comparison algorithm. The comparative results indicate that Gabor-FS algorithm is an effective iris recognition algorithm with high recognition rate. 


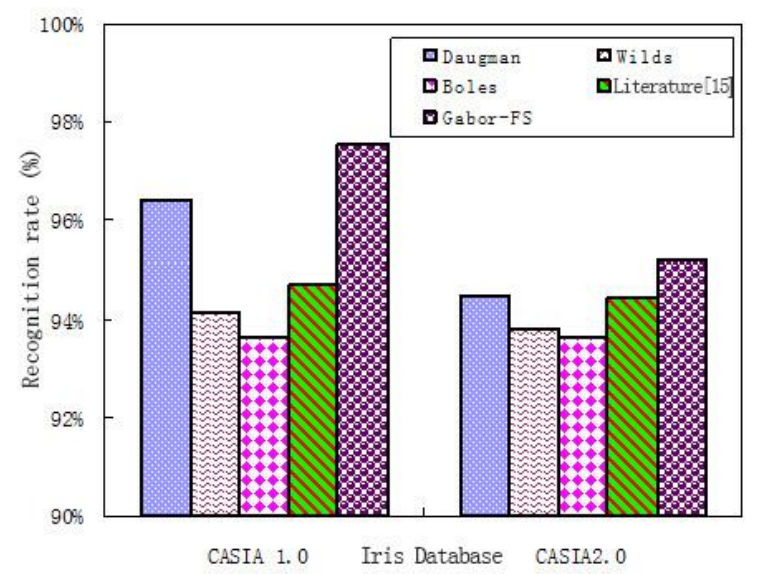

\section{Figure 7. Performance Comparisons with Classical Iris Recognition Algorithm}

\section{Conclusion and Next Step}

When using multi-scale multi-directional Gabor transform for the iris recognition process, the high dimensionality feature issue will occur. For this reason, this paper proposes an iris recognition algorithm based on Gabor-FS, tests the effectiveness of Gabor-FS through CASIA database, and discusses the effects of iris image block size and feature dimension on the algorithm performance. Experimental results show that it is a reasonable and effective attempt to adopt the iris recognition algorithm with the block idea and self-adaptive feature selection, which has certain advantages.

In the process of iris image recognition, the sub-block size correlates with the filter templates. The selection of filter parameters has certain influences on the recognition effect, which will be the direction for future research.

\section{Acknowledgements}

Thanks for Institute of Automation (Beijing), Chinese Academy of Sciences to provide CASIA iris database, so that the present study can be made smoothly.

\section{References}

[1] Z. Sun and T. Tan, "Ordinal Measures for Iris Recognition”, IEEE Trans. Pattern Analysis and Machine Intelligence, vol. 31, no. 12, (2009), pp. 2211-2226.

[2] Z. Luo and S. Lin, "Iris Image Preprocessing Method for Identity Authentication", South China University of Technology (Natural Science Version), vol. 37, no. 5, (2009), pp. 43-48.

[3] Q. Zheng and X. Li, "Rapid Iris Recognition Algorithm based on Gabor Filter", Computer Engineering and Design, vol. 32, no. 3, (2011), pp. 976-989.

[4] W. Yuan, Q. Feng and K. Li, 'Research on Iris Recognition Algorithm based on Single-frequency 2D-Gabor Filter", Optical Technique, vol. 36, no. 1, (2010), pp. 20-24.

[5] K. P. Hollingsworth, K. W. Bowyer and P. J. Flynn, "The Best Bits in an Iris Code", IEEE Transactions on pattern analysis and machine intelligence, vol. 31, no. 7, (2009), pp. 1-10.

[6] Y. Yin and C. Xu, "Iris Recognition Method based on Cross-power Spectrum", Chinese Journal of Image and Graphics, vol. 12, no. 5, (2007), pp. 854-859.

[7] P. Yao, X. Ye, W. Zhang, Z. Zhuang and B. Li, 'Iris Recognition Algorithm based on Improved Log-Gabor Wavelet", Computer-Aided Design and Computer Graphics, (2007), vol. 19, no. 5, pp. 563-568.

[8] X. Wang and D. Qinke, "An Iris Recognition Algorithm based on 2D-PLDA and Wavelet Sub-band", Chinese Journal of Image and Graphics, vol. 16, no. 1, (2011), pp. 59-64.

[9] Z. He, Lv Lintao, "Iris feature extraction and recognition based on ICA-MJE and SVM", Computer Applications, vol. 27, no. 6, (2007), pp. 1505-1507.

[10] N. Sun, W. Yu and M. Cao, "Iris Recognition Method based on PCA and ICA", Chinese Journal of Image and Graphics, vol. 13, no. 9, (2008), pp. 1701-1706. 
[11] Y. Duan and G. Liu, "Iris Recognition Method based on EMD and SVM", Computer Engineering and Applications, vol. 46, no. 30, (2010), pp. 188-190.

[12] L. Yan and Z. Biqing, "Iris Localization Method based on Regional Growth and Hough Transform", Computer Engineering and Applications, vol. 48, no. 8, (2012), pp. 200-202.

[13] Q. Jin, X. Tong and S. Bao, 'Research on Gabor Features Screening Oriented to Iris Recognition", Computer Engineering and Applications, vol. 28, no. 19, (2012), pp. 2101 -204.

[14] Institute of Automation, Chinese Academy of Sciences. CASIA Iris Image Database [EB/OL]. (2003-10)[2005-08]. http://www.Sinobiometrics.com.

\section{Author}

Jia Zhen Liang (1976.3-) Lecturer, Master, Research Orientation: Computing Network 
International Journal of Signal Processing, Image Processing and Pattern Recognition Vol. 8, No. 2 (2015) 